\title{
Norois
}

Environnement, aménagement, société

$236 \mid 2015$

Présence chinoise en Arctique, nautisme, ville-port, vulnérabilité, inondation

\section{La protection du bâti individuel et des commerces contre l'inondation. Opportunités et obstacles}

The adaptation of houses and shops to flooding. Opportunities and obstacles

Freddy Vinet, Frédéric Leone, Guillaume Lahache et Paul Cancel

\section{OpenEdition}

Journals

Édition électronique

URL : https://journals.openedition.org/norois/5723

DOI : $10.4000 /$ norois. 5723

ISBN : 978-2-7535-4945-6

ISSN : 1760-8546

Éditeur

Presses universitaires de Rennes

Édition imprimée

Date de publication : 30 décembre 2015

Pagination : 69-90

ISBN : 978-2-7535-4903-6

ISSN : 0029-182X

Référence électronique

Freddy Vinet, Frédéric Leone, Guillaume Lahache et Paul Cancel, « La protection du bâti individuel et des commerces contre l'inondation. Opportunités et obstacles », Norois [En ligne], 236 | 2015, mis en ligne le 30 décembre 2017, consulté le 01 février 2022. URL : http://journals.openedition.org/norois/ 5723 ; DOI : https://doi.org/10.4000/norois.5723 


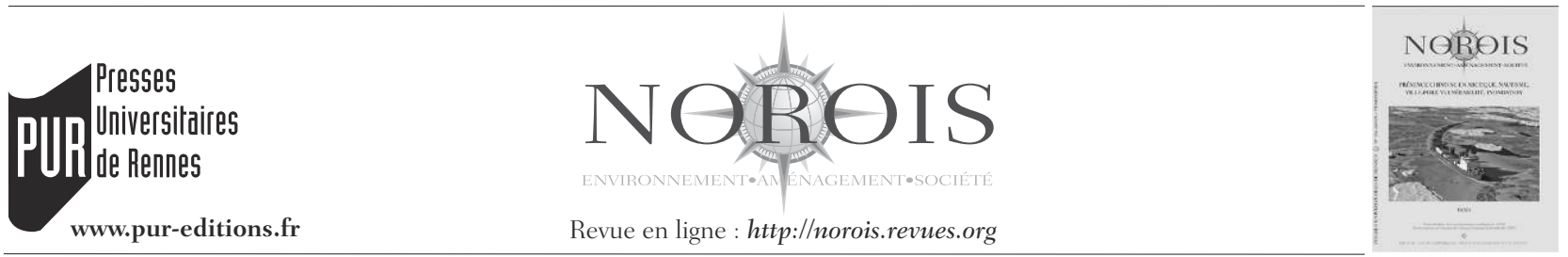

\title{
La protection du bâti individuel et des commerces contre l'inondation Opportunités et obstacles
}

\author{
The Adaptation of Houses and Shops to Flooding \\ Opportunities and Obstacles
}

\author{
Freddy VIneta ${ }^{a}$, Frédéric Leonea ${ }^{a}$, Guillaume Lahache ${ }^{b}$, Paul Cancel ${ }^{c}$
}

\footnotetext{
a UMR GRED IRD/Université Paul-Valéry Montpellier - route de Mende, 34199 MontPellier cedex 5. (freddy. vinet@univ-montp3.fr)(frederic.leone@univ-montp3.fr)

b Predictservices - 20 rue Didier-Daurat, 34170 CASTELnAu-Le-Lez (M2 GCRN 2013-2014). (guillaumelahache@ hotmail.fr)

`Quimper Communauté - Hôtel de Ville, CS 26004, 29107 Quimper Cedex. (paul.cancel@quimper.bzh)
}

Résumé : L'article propose un bilan des opportunités et des obstacles soulevés par l'adaptation des bâtiments au risque inondation. Cette question est devenue centrale dans les programmes de réduction du risque inondation. À partir d'une étude bibliographique et en s'appuyant sur une enquête approfondie sur la ville de Quimper, les auteurs mettent en relief la diversité des situations locales et la difficulté à aborder la réduction de la vulnérabilité des bâtiments par des mesures coercitives et générales. 290 habitations et 194 commerces ont été diagnostiqués sur la commune de Quimper afin de déterminer leur degré de vulnérabilité face à une inondation. Les occupants ont été questionnés sur leur degré de connaissance du risque et leur consentement à mettre en œuvre des mesures de protection de leur bâtiment. Sur ce dernier point, l'enquête montre que la mauvaise connaissance du risque n'est pas le seul facteur de désintérêt des habitants face à ce type de mesures. L'engagement des personnes est fortement lié à l'attachement au bien et aux facteurs qui en découlent (statut propriétaire/locataire, âge...). Les commerçants représentent une population difficile à mobiliser compte tenu du faible taux de propriétaires occupants et du renouvellement rapide des gérants.

\begin{abstract}
This paper proposes an assessment of the opportunities and obstacles in the adaptation of buildings to flood risk. It has become a key issue in flood risk reduction programs in which the reduction of vulnerability of building is a strong requirement. From a review of the existing documentation and relying on a thorough investigation in the city of Quimper, the authors highlight the diversity of local situations and the difficulty to address the reduction of the vulnerability of buildings through generic and coercive measures. 290 homes and 194 shops had been appraised in the city of Quimper according their vulnerability facing flooding. The occupants and shopkeepers had been questioned about their degree of knowledge of the risk and their willingness to implement the measures for the protection of their building. On the latter point, the survey shows that lack of knowledge of the risk is not the only factor of disinterest of the people facing this type of measure. The commitment of the people is strongly linked to attachment to the property and related factors such as ownership/renting, age... The shopkeepers are not easy to involve in flood risk reduction measures given the low rate of owner-occupiers and the rapid turnover of managers.
\end{abstract}

Mots clés : inondation - vulnérabilité - bâtiments - prévention - résilience

Keywords: flood risk - vulnerability - building - prevention - resilience 


\section{INTRODUCTION}

La réduction de la vulnérabilité des sociétés face aux catastrophes naturelles est une préoccupation qui a dépassé la seule sphère scientifique pour gagner les milieux gestionnaires et, dans le meilleur des cas, les politiques. Un certain nombre de mesures sont aujourd'hui préconisées pour réduire la vulnérabilité des personnes et des biens face aux inondations : pose de batardeaux, étage refuge, pose de sorties de toit pour évacuation par les airs, etc. En France, les plans de prévention des risques incluent de plus en plus ces mesures sous la forme de recommandations ou de prescriptions. Mais force est de constater que si les diagnostics progressent, la mise en œuvre systématique des mesures tarde. Au-delà de la caractérisation de la vulnérabilité du bâti, déjà largement traitée par la littérature technique et scientifique, il nous importe d'envisager la faisabilité des mesures d'adaptation individuelle des logements et des commerces et les conditions préalables à la mise en place d'une protection plus efficace des bâtiments, des biens et des personnes.

Les réflexions s'appuient sur des enquêtes et diagnostics effectués depuis cinq ans par les étudiants du master « gestion des catastrophes et des risques naturels » associés à l'UMR GRED de Montpellier dans différentes régions françaises (Haute-Loire, Alpes du Sud, île d'Oléron, Bretagne). Les éléments permettant d'expliquer les difficultés d'une réduction systématique de la vulnérabilité du bâti face à l'inondation sont semblables en dépit de situations locales diverses. Afin d'éviter la confusion des échantillons et des résultats, l'enquête effectuée en octobre 2013 dans le cadre du Master 2 « gestion des catastrophes et des risques naturels » à Quimper servira de support à cet article (Leone et Vinet, 2014).

Après une présentation des objectifs et du contexte général de la réduction de la vulnérabilité du bâti en France, nous exposerons les objectifs spécifiques de l'enquête menée à Quimper. Une première série de résultats concerne la caractérisation de la vulnérabilité des personnes, des logements et des commerces. On insistera ensuite sur les adaptations mises en place au niveau individuel, peu nombreuses mais suffisamment significatives pour prouver que cette adaptation est possible. Enfin nous terminerons sur une discussion autour des obstacles actuels et des conditions nécessaires à la généralisation de ces mesures d'adaptation.

\section{LA RÉDUCTION DE LA VULNÉRABILITÉ DU BÂTI : UNE PRIORITÉ AFFICHÉE DE LA PRÉVENTION}

\section{la réduction de la vulnérabilité : une préoccupation croissante des pouvoirs publics}

Il est maintenant admis au moins dans la communauté scientifique et chez les gestionnaires que les mesures structurelles telles que les digues ou les barrages ont des effets pervers et qu'elles ne doivent pas constituer le seul rempart contre les crues. L'accent est mis sur la réduction des vulnérabilités à la suite des injonctions internationales issues entre autres de la Conférence mondiale sur la prévention des catastrophes qui a adopté le «Cadre d'action de Hyogo pour 2005-2015 : Pour des nations et des collectivités résilientes face aux catastrophes ", cadre rediscuté lors de la conférence de Sendaï en mars 2015. En France, depuis 2011, les Programmes d'Action et de Prévention des Inondations (PAPI) intègrent obligatoirement un axe « réduction de la vulnérabilité ». Au sens large du terme, la réduction de la vulnérabilité a pour objectif d'agir sur les biens, les personnes, les territoires... afin de renforcer leur capacité de résistance face aux catastrophes et d'en diminuer les impacts. Ces mesures concernent aussi bien le renforcement du bâti que la préparation à la gestion de crise en passant par l'amélioration de la conscience du risque et la connaissance des gestes préventifs. Concernant le bâti existant en zone inondable, la réduction de la vulnérabilité vise à entreprendre des travaux plus ou moins lourds d'adaptation afin de limiter les dommages en cas de sinistre. Ces adaptations renforcent la résistance des bâtiments à l'eau, permettent la sauvegarde des populations (étage refuge, évacuation de toit) et des biens et facilitent la remise en état après un sinistre.

La réduction de la vulnérabilité de l'existant, c'està-dire des bâtiments actuellement en zone inondable reste un point faible de la prévention. Pour la France, le nombre d'habitants en zone inondable a été réévalué à plus de 17 millions dans l'EPRI 
(évaluation préliminaire du risque inondation) en 2011 (MEDDE, 2011) auxquels il faut ajouter le 1,4 million de personnes exposées aux submersions marines. Si l'on considère que le nombre moyen d'occupants par logement est d'environ deux personnes, les estimations les plus larges donnent donc quelque neuf millions de logements potentiellement inondables en France.

Les travaux des architectes (Kelman, 2002, 2007 ; Salagnac et Bessis, 2006) ou de géographes (Jousseaume et Mercier, 2009) ont montré le potentiel d'adaptabilité du bâti à la submersion. Il existe quelques études mettant en évidence l'efficacité de ces travaux notamment ceux cités par Torterotot (1993: 74-75). D'enquêtes effectuées sur Montauban, il ressort que l'adoption de mesures d'adaptation dépend du coût des mesures, de la solvabilité des personnes, du statut propriétaire ou locataire, du niveau d'aléa considéré et de l'âge des occupants... L'endommagement peut décroître de moitié si les mesures de prévention sont adaptées et «pour des submersions ne présentant pas un caractère exceptionnel ». De nouvelles évaluations sont en cours notamment dans le cadre du groupe de réflexion ACB/AMC (analyse coûtbénéfice/analyse multicritères) mis en place par le MEDDE afin de corroborer l'efficacité réelle de mesures comme la pose de batardeaux ou le changement des revêtements muraux. Les recherches de Thieken et al. (2005) sur l'Elbe après les inondations de 2002 sont riches d'enseignements en même temps qu'elles invitent à l'humilité. En effet, les auteurs ont comparé les dommages dans des habitations où des mesures de prévention avaient été prises avec ceux relevés dans des habitations n'ayant pas mis en œuvre de telles mesures. Il s'avère par exemple que le fait de mettre des biens en sécurité à l'étage diminue les dommages de quelques points de pourcentage. Idem pour l'arrimage des réserves d'hydrocarbures ou l'installation d'une pompe. C'est donc un ensemble de mesures et de comportement adaptés qui peut faire baisser significativement les dommages. Les auteurs notent que les dommages ne varient pas seulement en fonction des hauteurs d'eau ou de la vitesse de l'eau mais aussi en fonction du type d'enjeux et du comportement des occupants. Ceci est confirmé par des travaux récents notamment ceux de André (2013). Ce dernier montre d'après l'examen de dossiers d'expertise que
$80 \%$ des coûts de remise en état sont imputables au second œuvre (plâtrerie, cloisons, système électrique... p. 163). Il souligne également que les paramètres d'aléa combinés (hauteur d'eau, vitesse...) n'expliquent que $50 \%$ de la variance des dommages et donc que le type de bâtiment, la plus ou moins grande vulnérabilité de l'enjeu, expliquent une part importante de la variabilité des dommages.

Ainsi il y a tout lieu de croire que l'adaptation du bâti et des comportements des occupants sont un gisement préventif intéressant et un levier permettant à moyen et long terme une réduction des dommages.

\section{Un développement timide en France}

C'est le pari implicite des autorités françaises qui préconisent la prescription systématique de mesures (MEDD, 2004; MEDDE, 2012) dites de mitigation ${ }^{1}$ dans les Plans de Prévention des Risques (PPR). Depuis 2003, les PPRi incluent de plus en plus des recommandations (non obligatoires) ou des prescriptions (obligatoires) sur le bâti existant (logements et activités économiques). L'objectif est d'obliger ou d'encourager les propriétaires d'habitations, de commerces ou les industriels à engager des mesures concrètes afin de diminuer les dommages en cas de sinistre. Ces prescriptions sont parfois édictées de manière très fine comme dans le plan de prévention des risques de La Faute-sur-Mer/ L'Aiguillon-sur-Mer en Vendée où elles s'appuient sur une mesure de l'altitude du plancher de chaque bâtiment par un géomètre-expert. De plus, ces prescriptions sont éligibles aux subventions du fonds de prévention des risques naturels majeurs dit « fonds Barnier».

Toutefois, il faut reconnaître que ces mesures sont peu appliquées. Les services de l'État n'ont pas les moyens humains, juridiques et techniques d'en vérifier l'application. En 2011-2012, dans les départements de l'Hérault et de l'Aude, des appels ont été lancés par les services de l'État afin de proposer aux habitants concernés les subventions «fonds Barnier » promises. Il n'y eut que quelques demandes de dossiers. Sur les quinze expériences citées en exemple dans le guide édité

\footnotetext{
1. Ces mesures sont parfois appelées «mesures de mitigation ». Si cette réutilisation détournée d'un terme anglais n'est guère satisfaisante, elle a le mérite de la concision.
} 
par le MEEDDAT (2008), treize se sont arrêtées au niveau du diagnostic. Si l'on dispose en effet de nombreux diagnostics, les mises en œuvre restent très limitées et ponctuelles. Elles sont l'œuvre en général de collectivités territoriales volontaristes. On peut citer à cet égard le dispositif Alabri [http://www . alabri-smage.fr] mis en place dans le département du Gard depuis 2010.

\section{les mesures possibles}

Techniquement, les mesures sont exposées dans de nombreux guides et publications spécialisées (Crichton, 2003; Kreibich et al., 2005; CEPRI, 2010; MEDDE, 2012). Les agences nationales (FEMA, 1999; Certu-MEDD, 2004; DGUHC, 2005) et les instances de gestion des bassins hydrographiques (EPTB Saône et Doubs, 2009) ont publié successivement des guides depuis plus de quinze ans. Le type de mesure se décline suivant trois objectifs :

- réduire l'exposition des personnes et en facilitant les secours;
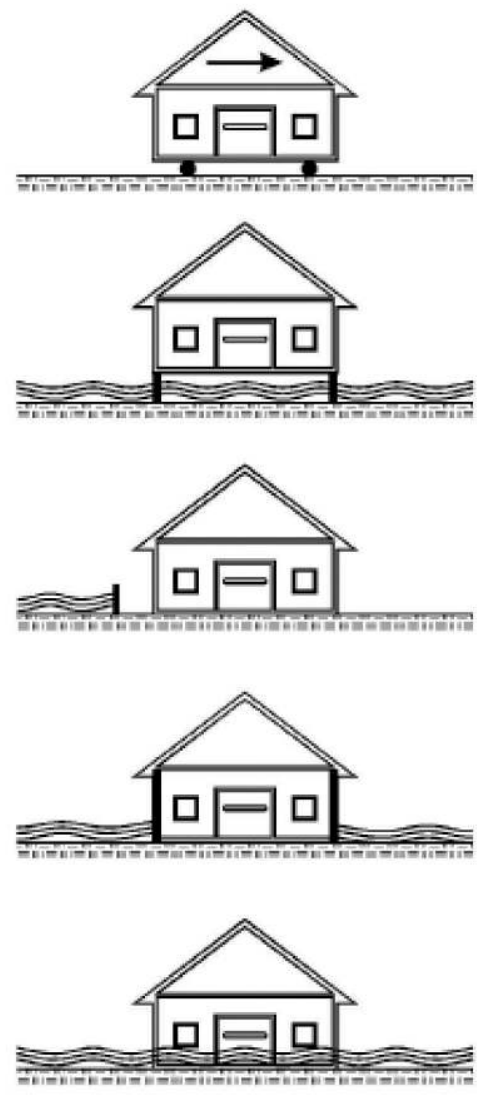

relocalisation dans un secteur non exposé

élévation du plancher de l'immeuble au dessus de la ligne d'eau

construction de murs (permanents ou temporaires) empêchant l'eau d'atteindre le bâtiment

Etanchéification externe empêchant l'eau de pénétrer dans le bâtiment (dry waterproofing)

Etanchéification interne limitant les dommages en cas d'inondation (wet waterproofing)
Figure 1 : Les approches de l'adaptation du bâti à l'inondation (adapté de Kreibich et al., 2005 et Andjelkovic, 2001)

The different approaches of building adaptation to flooding (adapted from Kreibich et al., 2005 et Andjelkovic, 2001) 
structures lorsque cette poussée cède en fin d'inondation. On distingue donc les techniques « en sec» destinées à étanchéifier le bâtiment et les techniques « en eau » pour lesquelles, l'eau étant impossible à contenir, il convient de limiter les dégâts à l'intérieur (bas de la figure 1). Par exemple, les batardeaux ne sont efficaces que sous certaines conditions. Leur hauteur doit être inférieure à $80 \mathrm{~cm}$ afin de pouvoir laisser passer les secours; de plus, au-delà de plusieurs jours de submersion, leur efficacité risque d'être mise à mal par des infiltrations d'eau venues d'autres sources comme les égouts non pourvus de clapets antiretour ou par les aérations.

Alors que les techniques sont bien connues et que les pratiques expérimentales existent en France et plus largement en Europe, on est en droit de se demander quels sont les blocages qui empêchent la généralisation de ces mesures. Plus simplement, pourquoi la réduction de la vulnérabilité des bâtiments existants face à l'inondation a-t-elle du mal à franchir le stade du diagnostic?

\section{L'ENQUÊTE LOCALE : CONTEXTE, OBJECTIFS ET MÉTHODOLOGIE}

\section{Contexte local : la ville de Quimper et l'inondation}

La ville de Quimper est exposée aux inondations de l'Odet et de ses affluents en particulier le Steïr qui conflue au cour de la ville. La ville est soumise aux submersions marines dans sa partie basse en aval de la confluence avec le Steïr. Les marées hautes de vives eaux engendrent des remontées d'eau dans les rues les plus basses (rue René Madec et place de la Terre-au-Duc).

Quimper a été particulièrement touché par les inondations en 1995 (figure 2) et en décembre 2000. Cette dernière crue majeure a causé 2,2 millions d'euros de dommages à la ville et près de 12 millions aux particuliers (SIVALODET, 2012). De nombreuses informations et photographies sur cette crue ont été mises en ligne par l'agglomération quimpéroise $^{2}$. Les inondations les plus récentes datent de l'hiver 2013-2014 mais n'ont pas atteint les niveaux de décembre 2000.

\footnotetext{
2. [http://sig-diffusion.quimper-communaute.fr/Internet/04/pli/PlanInondations.aspx? $p l=\& p 5=0 \& p 6=0]$.
}

La réduction de la vulnérabilité s'inscrit dans une longue série d'actions destinées à réduire le risque. L'histoire de la ville de Quimper a été jalonnée de mesures visant à contenir le risque inondation tout en souhaitant bénéficier de la présence des cours d'eau (Valy, 2010)

Récemment, depuis les inondations de 1995, la ville de Quimper a mis en place des mesures de protection collectives, l'amélioration ponctuelle de l'écoulement et un serveur «infocrues » destiné à avertir la population exposée en cas d'inondation prévue (tableau 1). L'usine Armorlux, installée dans le quartier de l'hippodrome et inondée en 1995 et 2000, a été délocalisée. L'espace a été rendu à l'écoulement et une digue protège le quartier de l'hippodrome des débordements les plus fréquents de l'Odet.

L'Odet a été l'un des premiers bassins-versants bretons à bénéficier en 2003 d'un programme d'action et de prévention des inondations (PAPI). Le PAPI 1 était orienté vers la finalisation de protections déjà engagées après les inondations de 1995. Le PAPI a été renouvelé en 2012 Il est orienté selon les 7 axes majeurs suivants (SIVALODET, 2013):

Axe 1 - Amélioration de la connaissance et de la conscience du risque;

Axe 2 - Surveillance, prévision des crues et des inondations ;

Axe 3 - Alerte et gestion de crise;

Axe 4 - Prise en compte du risque inondation dans l'urbanisme;

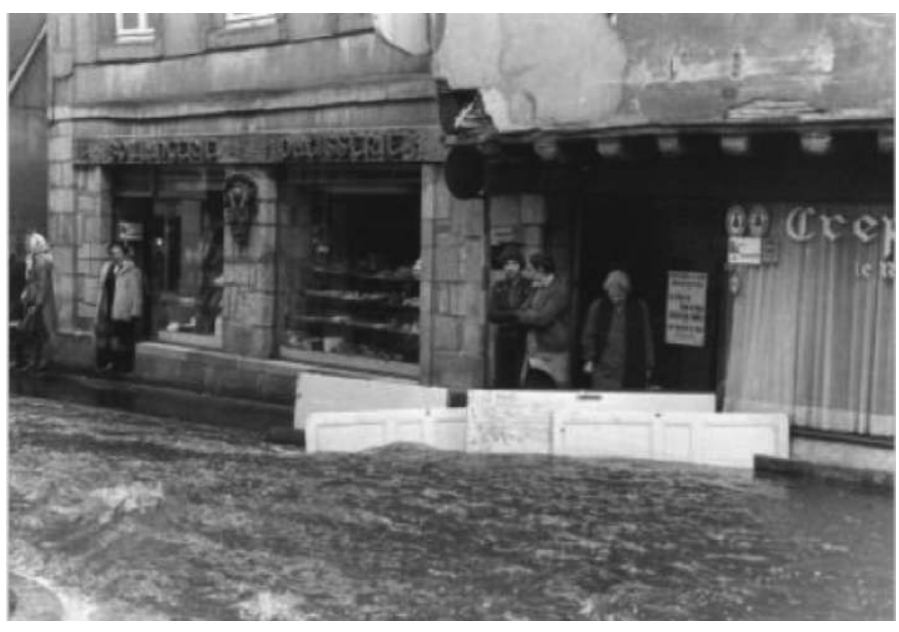

Figure 2 : Exemple de mesures de protection improvisées pendant la crue de 1995 (source : Mairie de Quimper)

Example of temporal adaptation measure taken during 1995 floods (source: Municipality of Quimper) 


\begin{tabular}{|c|c|}
\hline Année & Dispositif mis en place \\
\hline 1997 & Approbation du PPRi \\
2001 & Élaboration du « Schéma d'Aménagement et Gestion des Eaux (SAGE) (2001-2007) \\
2003 & Instauration du PAPI 1 sous l’impulsion du SIVALODET \\
2004 & Révision du PPRi suite aux inondations de 2000 \\
2007 & Approbation et mise en \\
2007 & œeuvre du SAGE (2007-2010) \\
2009 & Nouvelle révision du PPRi \\
2009 & Élaboration du «Plan Communal de Sauvegarde » (PCS) et du DICRIM \\
2010 & Mise en place du dispositif d'alerte Info-Crues \\
2012 & Révision du SAGE (2010-2014) \\
\hline
\end{tabular}

Tableau 1 : Historique de la gestion du risque et dispositifs mis en place sur la commune de Quimper depuis les inondations de 1995. Flood management measures implemented on the municipality of Quimper since 1995

Axe 5 - Actions de réduction de la vulnérabilité des personnes et des biens;

Axe 6 - Ralentissement des écoulements;

Axe 7 - Gestion des ouvrages de protection hydraulique.

L'enquête présentée dans cet article entre dans le cadre des actions des axes 1 et 5 du PAPI.

Quimper est par ailleurs soumis à un plan de prévention des risques inondation (figure 3 planche I) approuvé en 1997, révisé en 2004 pour intégrer la crue de 2000 dans l'aléa et révisé à nouveau en 2007 pour prendre en compte le projet de réaménagement du secteur de la Providence par la ville de Quimper. Le Plan de prévention des risques ne contient pas de prescriptions sur l'habitat existant.

\section{Objectifs}

L'objectif premier de l'enquête était de diagnostiquer la vulnérabilité du bâti et des habitants face au risque inondation. Les objectifs de second ordre, mais aussi importants en termes stratégiques pour les collectivités territoriales impliquées, étaient de déterminer les potentialités et les freins éventuels à la mise en place de protections individuelles et d'adaptations du bâti face aux inondations. Au-delà des objectifs opérationnels, la richesse des informations collectées alimente le débat sur l'opportunité et la faisabilité de ces mesures et sur l'efficacité de la prévention du risque inondation en général.

\section{Contenu et réalisation des diagnostics}

Deux diagnostics ont été élaborés. L'un concerne les logements privatifs. Il comporte 101 questions ou « entrées ». Le diagnostic « commerce » comprend 119 entrées.

Le tableau 2 décrit les thèmes abordés dans le diagnostic « habitat » et les modalités de collecte des informations. Globalement, les questions sont d'ordre factuel notamment celles qui constituent le corps du diagnostic à savoir la vulnérabilité du bâtiment (revêtement de sol, de mur, système électrique...). Ces éléments sont descriptifs et souffrent peu des biais classiques rencontrés dans les passations d'enquêtes. Pour ces éléments diagnostiqués, l'enjeu pour les enquêteurs était surtout d'avoir accès aux locaux.

Le diagnostic « commerce » comprend, outre les thèmes énumérés dans le tableau 2, des questions sur la vulnérabilité économique et humaine du commerce notamment en qualifiant la présence de stocks, les matériaux d'exploitation et en caractérisant la présence humaine (nombre de personnes en employés/clientèle, heures d'ouverture...).

Les diagnostics ont été effectués du 21 octobre au 4 novembre 2013. Ils ont été remplis lors d'entrevues en face-à-face entre les binômes d'enquêteurs et les occupants des logements et des commerces. La période comprenait un week-end, des jours ouvrables et des vacances scolaires (Toussaint) ce qui permit de multiplier les occasions de rencontrer les occupants. Lorsqu'un logement ou com- 


\begin{tabular}{|c|c|c|c|}
\hline Thèmes principaux & Descriptif du thème & Numéros de question & $\begin{array}{l}\text { Mode d'acquisition } \\
\text { des informations }\end{array}$ \\
\hline $\begin{array}{l}\text { Éléments de contexte et } \\
\text { identification du logement } \\
\text { diagnostiqué }\end{array}$ & $\begin{array}{l}\text { - localisation, type de loge- } \\
\text { ment. } \\
\text { - date de passage, } \\
\text { - réceptivité de l'occupant } \\
\text { (refus...) }\end{array}$ & Questions 1 à 10 & Remplis par enquêteur \\
\hline $\begin{array}{l}\text { Connaissance et conscience } \\
\text { du risque } \\
\text { Connaissance mesures de } \\
\text { prévention }\end{array}$ & $\begin{array}{c}\text { - connaissance du risque } \\
\text { - Connaissance PPR, système } \\
\text { alerte crue } \\
\text { - sentiment de menace }\end{array}$ & Questions 11 à 21 & $\begin{array}{l}\text { Questions ouvertes et fermées } \\
\text { à(ux) occupant(s) }\end{array}$ \\
\hline $\begin{array}{l}\text { Qualification de l'aléa à } \\
\text { l'échelle du bâti }\end{array}$ & $\begin{array}{c}\text { - nombre et date des inonda- } \\
\text { tions vécues dans ce logement, } \\
\text { hauteurs d'eau atteintes, } \\
\text { impacts }\end{array}$ & Questions 22 à 33 & $\begin{array}{l}\text { Questions majoritairement } \\
\text { fermées à(ux) occupant(s) }\end{array}$ \\
\hline Inondation de décembre 2000 & - vécu de l'inondation de 2000 & Questions 34 à 41 & $\begin{array}{c}\text { Questions ouvertes et fermées } \\
\text { à(ux) occupant(s) }\end{array}$ \\
\hline Vulnérabilité structurelle & $\begin{array}{l}\text { - caractérisation de la struc- } \\
\text { ture du bâtiment, du second } \\
\text { œuvre, des ouvertures, des } \\
\text { revêtements sol et mur, du sys- } \\
\text { tème électrique }\end{array}$ & Questions 42 à 81 & $\begin{array}{c}\text { Diagnostic effectué par } \\
\text { les enquêteurs avec le(s) } \\
\text { occupant(s) }\end{array}$ \\
\hline $\begin{array}{c}\text { Réalisation d'aménagements } \\
\text { préventifs }\end{array}$ & $\begin{array}{l}\text { - Aménagements existants } \\
\text { - Consentement à mettre en } \\
\text { œuvre des mesures d'adapta- } \\
\text { tion }\end{array}$ & Questions 82 à 94 & $\begin{array}{l}\text { Questions ouvertes à(ux) } \\
\text { occupant(s) }\end{array}$ \\
\hline $\begin{array}{c}\text { Caractérisation des occupants } \\
\text { du bâtiment/vulnérabilité } \\
\text { humaine }\end{array}$ & $\begin{array}{l}\text { - nombre de personnes, sexe, } \\
\text { âge, profession } \\
\text { - présence enfant ou handi- } \\
\text { capé } \\
\text { - année occupation logement } \\
\text { - propriétaire/locataire }\end{array}$ & Questions 94 à 101 & $\begin{array}{l}\text { Questions fermées à(ux) } \\
\text { occupant(s) }\end{array}$ \\
\hline
\end{tabular}

Tableau 2 : Présentation du diagnostic « habitat » Description of the survey "housing"

merce n'était pas accessible, les deux enquêteurs repassaient deux fois à des horaires ou jours différents de façon à multiplier les probabilités de rencontrer les occupants. Lorsque les personnes étaient présentes mais indisponibles, un rendezvous était pris. Des renseignements informels de voisinage permettaient également de savoir si un logement ou un commerce était occupé ou non (cas de personnes en séjour prolongé à l'hôpital...). Les répondants se répartissent entre 142 hommes et 148 femmes. À plusieurs reprises, les entrevues se sont passées en présence de deux personnes. Dans ce cas, les enquêteurs ont veillé à ce que, pour les questions relevant de la perception ou de l'opinion, ce soit un répondant et toujours le même qui réponde. Faute de temps, il n'était pas possible d'étendre les questions à tous les occupants de l'habitation ou du commerce. Pour celles qui relevaient du diagnostic technique, les personnes accompagnant le répondant principal (mari ou femme) pouvaient être prises en compte.

\section{Champ géographique de l'étude, modalités de l'enquête et échantillon}

La volonté de la municipalité quimpéroise était de limiter l'enquête au périmètre de l'inondation du 13 décembre 2000 dont la période de retour est estimée à 40 à 50 ans dans la ville de Quimper sur les rivières Odet et Steïr (figure 4 - planche II). La population cible totale est constituée par l'ensemble des commerces et des habitations situés dans l'enveloppe de la crue de décembre 2000. Malgré plusieurs demandes auprès de la municipalité de Quimper, il 
n'existe pas de modélisation hydraulique reconstituant la crue de 2000 ni les crues Q100 ou Q50. D'autres données hydrologiques existent ( $c f$. les travaux de J. Valy, 2010) mais elles ne nous ont pas été communiquées. Nous ne disposions lors des diagnostics que de l'enveloppe de la zone inondée en 2000.

La limitation au périmètre inondé en 2000 a permis d'enquêter des logements et commerces que l'on savait avoir été touchés par cet événement et ainsi de mesurer la préservation de la mémoire chez les occupants actuels et le renouvellement des populations résidentes, deux variables clés de l'enquête. Ce sont 654 enjeux enquêtables (commerces et logements) qui ont été identifiés dans l'enveloppe de la crue de décembre 2000 dans la ville de Quimper à partir de la BD topo IGNC et d'un premier recensement des enjeux effectué par le SIVALODET, remis à jour sur le terrain par le groupe d'enquêteurs. Le diagnostic s'est fait exclusivement en présentiel avec une durée moyenne des diagnostics de trente minutes ce qui limite les biais liés aux autodiagnostics. 90 logements ou commerces n'ont pu être diagnostiqués suite à l'absence des occupants (personnes en vacances, hospitalisées, logement ou commerces vacants...). 80 n'ont pu l'être suite au refus de l'occupant de laisser accès à son logement et de répondre aux questions. Les logements situés en zone inondable mais non inondables du fait de leur hauteur (logement à l'étage dans immeuble) ont été exclus de l'enquête (tableau 3 et figure 5).

Sur la population cible totale de 654 logements et commerces inondables dans l'enveloppe de crues de 2000, 484 diagnostics exploitables ont été récoltés soit un taux de couverture de $74 \%$. Le taux de refus a été de $12 \%$. Ces 484 diagnostics se répartissent en 290 logements et 194 commerces. Les résultats ont été exploités dans une base de données Excel (C) et dans le logiciel de traitement Sphinx® puis intégrés dans une base de données géoréférencées à chaque polygone « enjeu » (logement ou commerce).

L'enquête est extrêmement riche d'enseignements et il ne saurait être question d'exposer l'ensemble des résultats. Nous avons choisi pour cet article de centrer notre propos sur trois points complémentaires. Il s'agissait tout d'abord de caractériser la vulnérabilité du bâti et son degré d'adaptation (ou d'inadaptation) à l'inondation. Nous avons ensuite recherché des facteurs explicatifs à l'inadaptation

\begin{tabular}{|c|c|c|c|c|c|}
\hline diagnostics & $\begin{array}{c}\text { Logements et } \\
\text { commerces } \\
\text { enquêtables }\end{array}$ & $\begin{array}{c}\text { Logements et } \\
\text { commerces non } \\
\text { enquêtés }\end{array}$ & $\begin{array}{l}\text { Logements } \\
\text { et commerces } \\
\text { soumis à } \\
\text { l'enquête }\end{array}$ & Refus & $\begin{array}{c}\text { Nombre } \\
\text { d'enquêtes } \\
\text { exploitables }\end{array}$ \\
\hline Quartier Hippodrome & 134 & $25(19 \%)$ & $109(81 \%)$ & $9(7 \%)$ & $100(75 \%)$ \\
\hline $\begin{array}{l}\text { Quartier du Moulin Vert } \\
\text { centre }\end{array}$ & 79 & $0(0 \%)$ & $79(100 \%)$ & $16(20 \%)$ & $63(80 \%)$ \\
\hline Quartier de la Providence & 57 & $13(23 \%)$ & $44(77 \%)$ & $3(5 \%)$ & $41(72 \%)$ \\
\hline Quartier Frout & 38 & $5(13 \%)$ & $33(87 \%)$ & $4(11 \%)$ & $29(76 \%)$ \\
\hline $\begin{array}{l}\text { Quartier Centre- } \\
\text { Préfecture }\end{array}$ & 41 & $3(7 \%)$ & $38(93 \%)$ & $4(10 \%)$ & $34(83 \%)$ \\
\hline Quartier Moulin Vert Sud & 50 & $8(16 \%)$ & $42(84 \%)$ & $7(14 \%)$ & $35(70 \%)$ \\
\hline $\begin{array}{l}\text { Quartier Moulin Vert } \\
\text { Nord }\end{array}$ & 28 & $1(4 \%)$ & $27(96 \%)$ & $9(32 \%)$ & $18(64 \%)$ \\
\hline Quartier Gare & 28 & $6(21 \%)$ & $22(79 \%)$ & $1(4 \%)$ & $21(75 \%)$ \\
\hline Quartier Hyper Centre & 88 & $5(6 \%)$ & $83(94 \%)$ & $13(15 \%)$ & $70(80 \%)$ \\
\hline Quartier Pont Firmin & 64 & $21(33 \%)$ & $43(67 \%)$ & $9(14 \%)$ & $34(53 \%)$ \\
\hline $\begin{array}{l}\text { Quartier concerné par la } \\
\text { Submersion Marine }\end{array}$ & 47 & $3(6 \%)$ & $44(94 \%)$ & $5(11 \%)$ & $39(83 \%)$ \\
\hline Totaux & $654(100 \%)$ & $90(14 \%)$ & $564(86 \%)$ & $80(12 \%)$ & $484(74 \%)$ \\
\hline
\end{tabular}

Tableau 3 : Taux de réalisation des diagnostics (logement et commerces) par quartiers Response rate at the survey according the different districts 


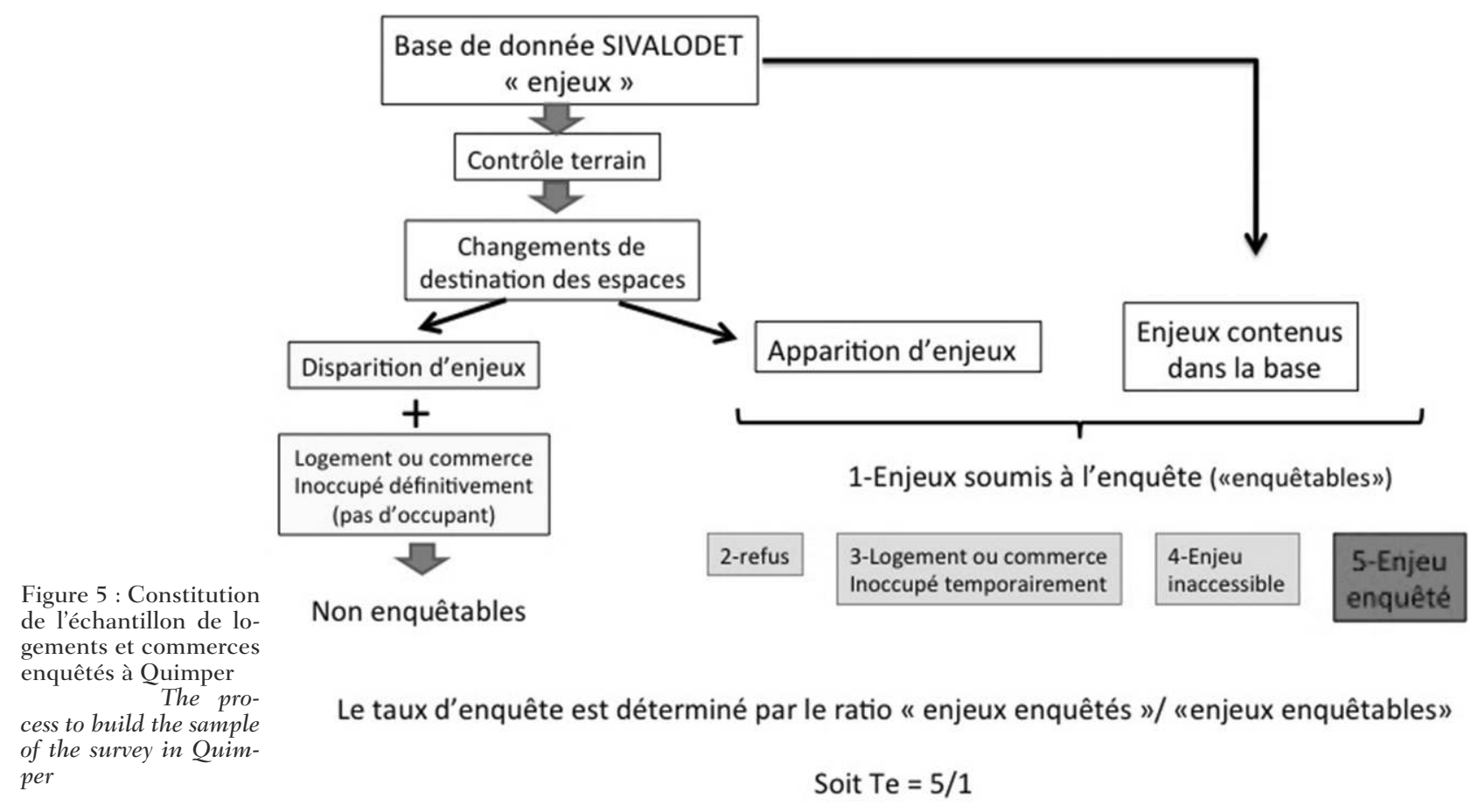

du bâti ou au contraire à l'adoption de mesures préventives par les occupants en étudiant le cas particulièrement difficile des commerces. In fine, le but de cet article est de mettre en exergue les blocages empêchant la mise en place généralisée de mesures d'adaptation et, en contrepoint, les prérequis nécessaires à cette mise en place.

\section{L'ADAPTATION/INADAPTATION DU BÂTI À L'INONDATION : ÉLÉMENTS QUANTITATIFS ET EXPLICATIFS}

\section{L'adaptation du bâti à l'inondation est inégale et le plus souvent fortuite}

L'adaptation/inadaptation du bâti à l'inondation a été analysée à partir de treize critères généraux (tableau 4).

Il faut, dans l'adaptation/inadaptation du bâti, distinguer les caractéristiques générales du bâtiment, soit dans sa structure (étage, sortie de toit) soit dans le second œuvre (prises électriques...) qui sont issues de la période de construction et les mesures spécifiques contre l'inondation adoptées postérieurement à la construction et qui peuvent donc révéler une adaptation plus « volontariste » au risque.

\section{Accès à un étage}

Pour la première série de critères, on observe par exemple que de $31 \%$ des habitations n'ont pas d'accès à l'étage soit dans une maison (maison de plainpied) soit dans un immeuble. Pour les commerces, ce taux passe à $69 \%$ ce qui souligne la grande vulnérabilité des commerces notamment en centre-ville, nous y reviendrons.

\section{Réseau électrique}

Le réseau électrique est un critère important dans l'évaluation de la vulnérabilité structurelle du bâti en zone inondable. Particulièrement fragile, sa remise en état ou son remplacement sont coûteux et longs. Le ministère de l'Écologie, du Développement Durable et de l'Energie recommande de « positionner les tableaux électriques et les différents équipements électriques au-dessus du niveau de la crue de référence ou des plus hautes eaux connues » (MEDDE, 2012, p. 50). Ainsi à Quimper, $54 \%$ des logements situés en zone inondée 2000 ont des prises électriques situées à une hauteur inférieure ou égale à $25 \mathrm{~cm}$ du sol, ce qui compromet la sécurité du réseau électrique. 


\begin{tabular}{|l|}
\hline Critères généraux \\
\hline État des murs extérieurs \\
Nombre d'étage \\
Nombre d'ouvertures (portes et fenêtres) en RDC \\
Nombre d'aérations \\
Bâti sujet aux remontées d'eau par les canalisations \\
Hauteur des prises, du compteur électrique, \\
du disjoncteur au RDC \\
Matériaux des murs au RDC \\
Matériaux de la porte principale au RDC \\
Présence d'un vide sanitaire \\
\hline Mesures spécifiques \\
\hline Présence de clapets anti-retour sur les canalisations \\
Présence de batardeaux \\
Revêtement des murs \\
Revêtement des sols \\
\hline
\end{tabular}

Tableau 4 : Critères d'adaptation/inadaptation du logement ou commerce à l'inondation

flooding

Criteria to assess adaptation of houses and shops to

Globalement, l'adaptation/inadaptation du bâti à l'inondation est un phénomène fortuit, c'est-àdire lié avant tout aux règles en vigueur au moment de la construction du logement ou du commerce. L'adaptation constatée du bâti à l'inondation ne signifie pas que cette adaptation ait été conçue initialement à cette intention. La présence d'un étage correspond à des règles d'urbanisme - par exemple un coefficient d'occupation des sols élevé - qui n'ont pas de finalité préventive.

Car si l'on se réfère aux mesures spécifiques de protection contre les inondations, au premier rang desquelles la présence de batardeaux, seuls 15 habitations $(5 \%)$ et 17 commerces $(8,7 \%)$ en sont actuellement équipés. Il en va de même des clapets antiretour des eaux usées. Si $24 \%$ des habitants estiment que, lors des inondations, l'eau remonte par leur système de canalisations, seuls $5 \%$ des habitants interrogés sont en mesure d'affirmer que leur logement en est équipé et $58 \%$ des personnes interrogées ne répondent pas à la question.

Les taux d'équipement sont très inégaux selon les quartiers mais nous avons pu identifier un quartier, celui de l'hippodrome, où l'adaptation du bâti et la conscience du risque sont particulièrement présents. 
le Steïr. En comparaison, seuls $35 \%$ des habitants et $42 \%$ des commerçants ayant emménagé après la crue de 2000 se sentent menacés par ces cours d'eau. Le vécu des inondations influence logiquement la prise de mesures de précaution. 147 personnes sur 290 interrogées ont vécu au moins une inondation, la plupart celle de 2000. Le taux de personnes se disant favorable à la mise en place de mesures de protection individuelles est de $51 \%$ dans cet échantillon contre $29 \%$ dans l'échantillon total logement.

Enfin, même lorsque les mesures sont présentes, les comportements préventifs ne sont pas transmis entre les bailleurs d'une part et les locataires et gérants de commerce d'autre part. La présence de certains équipements d'adaptation au risque inondation ne signifie pas qu'ils soient opérationnels en temps voulu comme en ont témoigné certains locataires.

\section{La question des hauteurs d'eau : une expertise supplémentaire nécessaire}

L'adaptation du bâti à l'inondation doit se faire en lien étroit avec les données d'aléa. Il n'existe pas à notre connaissance de modélisation hydraulique reconstituant différentes enveloppes de la crue de 2000 ni les crues Q100 ou Q50. Eussent-elles été disponibles, ces sorties de modélisation hydraulique auraient été calculées par rapport au terrain naturel ce qui est d'aucune utilité pour préparer la mise en place de mesure d'adaptation du bâti (CEPRI, 2010). Il convient en effet de juger de la pertinence des mesures d'adaptation (batardeau, étage refuge...) par rapport à la hauteur d'eau atteinte dans le bâtiment.

La hauteur d'eau atteinte dans le bâtiment lors de crues passées a été recueillie pour 118 logements et 81 commerces. Elle atteint deux mètres au maximum. Ces hauteurs sont très hétérogènes d'un bâtiment à l'autre en fonction de la hauteur du plancher et ne peuvent être exploitées cartographiquement. Ce ne sont que des indications vraiment propres à chaque bâtiment. Si des mesures devaient être mises en œuvre dans les bâtiments, elles devraient se référer à la hauteur du plancher et à la hauteur d'eau dans le bâtiment et non à la hauteur d'eau sur terrain naturel qui est généralement fournie par les modèles hydrauliques. Ainsi, les données de hau-

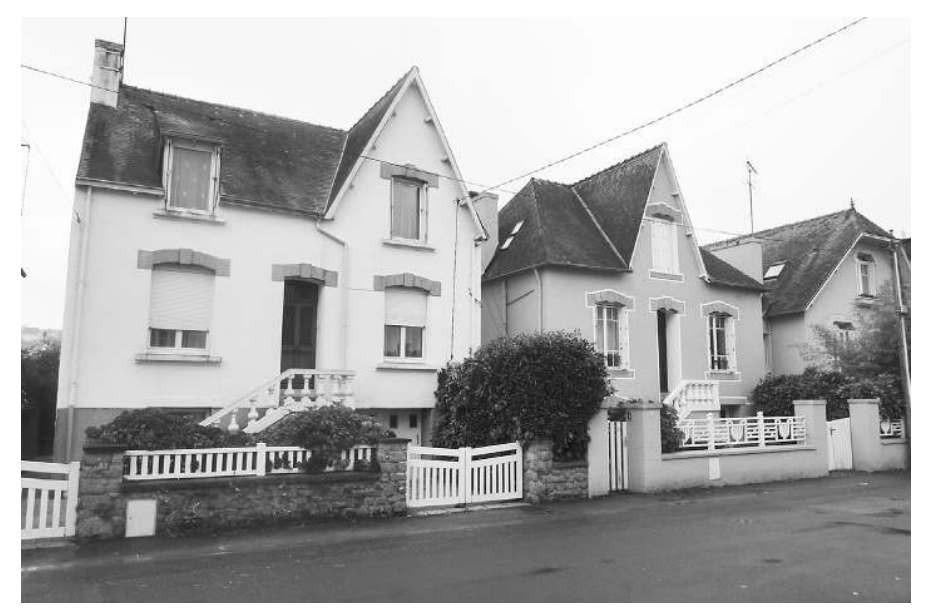

Figure 6 : Les maisons à étage sur rez-de-chaussée non habité dans le quartier de l'Hippodrome

Multiple-storey houses in the Hippodrome district in Quimper

teur d'eau collectées lors des diagnostics seront précieuses lorsqu'elles pourront être mises en relation avec la hauteur du plancher déterminée par expertise géomètre.

\section{LES OBSTACLES À LA GÉNÉRALISATION DES MESURES D'ADAPTATION DU BÂTI}

À la lumière du cas quimpérois et en s'appuyant sur la bibliographie existante, on peut dégager un certain nombre de facteurs favorables ou défavorables à la prise de mesures de réduction de la vulnérabilité du bâti. Plus que techniques ou financiers, les obstacles sont avant tout psychosociologiques.

\section{Les obstacles à la généralisation des mesures de protection individuelles}

181 personnes sur 290 interrogées disent connaitre des mesures de protection mais à la question «seriez-vous prêt à mettre en place des mesures de réduction de la vulnérabilité », ils ne sont que 85 (sur 290 soit $29 \%$ ) à répondre par l'affirmative. Plus intéressants sont les motivations des refus. 116 personnes ont précisé ce qui les dissuadait de mettre en place ces mesures (figure 7). Entre la confiance en d'autres mesures de prévention, la déresponsabilisation des locataires ou/et des personnes âgées... les raisons invoquées pour ne pas mettre en place des mesures d'adaptation du bâti sont multiples. Les réponses (libres) peuvent être classées en 4 registres. Le premier englobe les 52 réponses 
dénotant une déresponsabilisation (fondée ou infondée). Les raisons en sont diverses. Outre les 18 répondants qui répondent sans plus de précisions qu'ils ne sont pas concernés, les autres invoquent leur âge (9 d'entre eux), pour les locataires, la responsabilité du propriétaire (10 réponses), un départ prochain du logement (5)... La prise en charge des dommages par l'assurance n'est évoquée que par 4 personnes sur 116. Le second registre concerne les personnes qui ne se sentent pas menacées soit qu'elles ont confiance en d'autres moyens de prévention (alerte, digues...) soit qu'elles estiment que ce n'est pas nécessaire (sans plus de précision) auxquelles il faut ajouter les 8 répondants qui ont déjà installé les équipements suffisants. Un troisième groupe de réponse concerne le coût des mesures mais cet argument n'est invoqué que par 15 personnes (sur 116) et enfin 20 personnes soulignent l'inefficacité des mesures.

\section{Conscience et connaissance du risque}

La connaissance de l'aléa et une conscience du risque sont souvent invoquées pour expliquer la motivation des personnes exposées à adapter leur logement (Colbeau-Justin et al., 2003; Aviotti A., 2014). Les personnes aménageraient leur logement car elles auraient conscience du risque et aurait connaissance du caractère inondable de la zone. Selon Verrhiest et Guézo (2006) la volonté d'agir ressort du niveau de connaissance de l'aléa-conscience du risque - et de la valeur donnée au risque par rapport aux autres enjeux ou contraintes - acceptation $d u$ risque. C'est sans doute une condition nécessaire mais insuffisante pour faire émerger une culture de prévention individuelle. Du fait de la relative fréquence des inondations, de la proximité visuelle des cours d'eau et des crues relativement récentes (2000) la conscience du risque est relativement forte à Quimper. À la question «pensez-vous être en zone inondable? », $91 \%$ des commerces soumis à l'enquête et $89 \%$ des habitants concernés par l'enquête répondent « oui » et ont des éléments de connaissance sur l'aléa.

De même, le vécu des inondations et la conscience du risque sont un socle préalable à la mise en place de mesures d'adaptation du bâti comme l'a montré le cas du quartier de l'Hippodrome. D'ailleurs, il serait vain de vouloir mettre en place ces mesures là où n'existe aucune conscience du risque. La conscience du risque dont fait partie le travail de mémoire n'est pas une fin en soi ; elle est un terreau préalable à l'instauration de mesures de réduction du risque. registre : «non concerné»

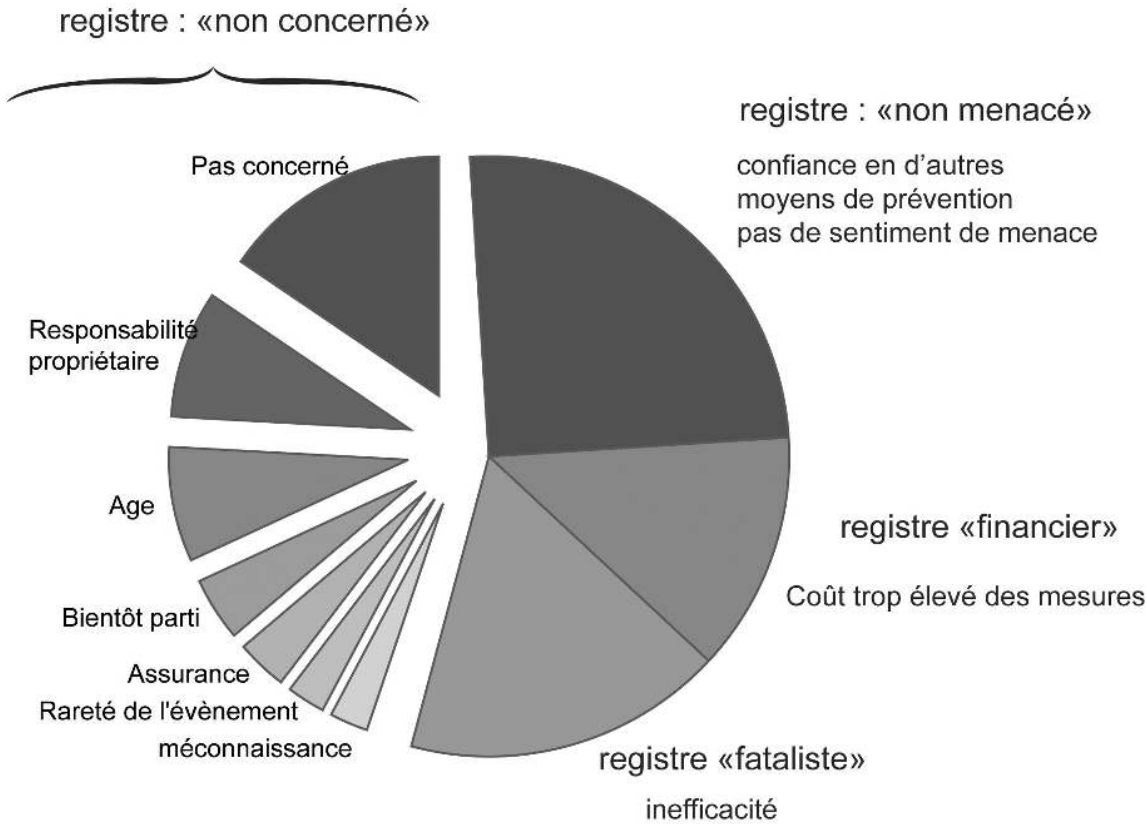

Figure 7 : Motivations des refus à vouloir mettre en place des mesures de mitigation (Source : enquêtes terrain Quimper 2013)

Motivations of people who refuse adaptation measures (Source: field survey, 2013) 


\section{La connaissance des mesures de mitigation}

Plus que la conscience du risque, la méconnaissance des mesures d'adaptation possibles semble être un obstacle. $37 \%$ des habitants et $38 \%$ des commerçants interrogés ne connaissent pas de mesures de protection individuelles contre les inondations.

Les questions relatives au consentement à payer ont été un relatif échec. À la question « combien seriez-vous prêts à investir pour des mesures de réduction de la vulnérabilité de votre logement? ", seules 75 personnes $(26 \%)$ ont répondu parmi lesquelles 57 cochent la somme de moins de 1000 euros. Ceci est compréhensible, les personnes n'ont pas connaissance des mesures possibles ni encore moins de leur coût ou des gains réalisables en termes de dommages évités. Ainsi, et c'est sans doute une des conclusions majeures de cette étude, avant d'engager des campagnes de mise en place de mesures, il convient de déployer un travail pédagogique avec des expositions, des démonstrations in-situ... afin de montrer la gamme des mesures d'adaptation, les arguments financiers (taux de subvention) venant après.

\section{Âge et consentement à adapter l'habitation}

Lorsque l'on met en relation le consentement à mettre en place des mesures de réduction de la vulnérabilité avec l'âge des répondants, les effectifs se distribuent en trois classes (tableau 5).

Les réponses positives sont surreprésentées dans les classes d'âge «adulte » (26-60 ans). Le « Non » est plus fréquent dans les réponses des personnes âgées de moins de 25 ans et de plus de 60 ans. La relation est significative pour un seuil minimal de $3 \%$.

\section{L'attachement au bien}

Ce qui ressort finalement, c'est que plus que la connaissance et le degré d'information sur le risque, c'est l'attachement au bien qui est la valeur cardinale déterminant la volonté d'investissement des occupants dans la protection de leur bien. C'est flagrant lorsque l'on compare propriétaires-occupants et locataires. Ainsi, $8 \%$ des propriétaires contre $18 \%$ des locataires déclarent ne pas savoir ou ne pas être en zone inondable. De plus, $35 \%$ des locataires déclarent ne pas avoir été informés sur le risque inondation contre $6 \%$ des propriétaires-occupants.

\begin{tabular}{|c|c|c|c|c|c|c|}
\hline \multicolumn{7}{|l|}{ Effectifs Observés } \\
\hline & $19-25$ ans & $26-45$ ans & $46-60$ ans & $61-75$ ans & $>75$ ans & total \\
\hline OUI & 2 & 33 & 24 & 21 & 5 & 85 \\
\hline $\mathrm{NON}$ & 11 & 64 & 45 & 45 & 40 & 205 \\
\hline Total & 13 & 97 & 69 & 66 & 45 & 290 \\
\hline \multicolumn{7}{|c|}{ Effectifs Théoriques (indépendance) } \\
\hline & $19-25$ ans & $26-45$ ans & $46-60$ ans & $61-75$ ans & $>75$ ans & Total \\
\hline OUI & 3,81 & 28,43 & 20,22 & 19,34 & 13,19 & 85 \\
\hline NON & 9,19 & 68,57 & 48,78 & 46,66 & 31,81 & 205 \\
\hline Total & 13 & 97 & 69 & 66 & 45 & 290 \\
\hline \multicolumn{7}{|c|}{ Différences Effectifs Observés - Effectifs Théoriques } \\
\hline & $19-25$ ans & $26-45$ ans & $46-60$ ans & $61-75$ ans & $>75$ ans & \\
\hline OUI & $-1,81$ & 4,57 & 3,78 & 1,66 & $-8,19$ & \\
\hline NON & 1,81 & $-4,57$ & $-3,78$ & $-1,66$ & 8,19 & \\
\hline
\end{tabular}

Valeur du $\mathrm{KHI}^{2}=10,65$

Seuil de significativité critique du $\mathrm{KHI}^{2}=3 \%$

Tableau 5 : Relation entre l'âge des répondants et le consentement à mettre en place des mesures d'adaptation (Question : « Seriez-vous prêt à mettre en place des mesures de réduction de la vulnérabilité ? »)

Age and willingness to implement adaptation measures on houses 
On soulignera que le fait de déclarer ne pas avoir été informé ne signifie pas qu'une information n'a pas été effectuée lors de l'IAL (information acquéreurs-locataires) par exemple ou lors des campagnes d'informations de la municipalité dans le cadre du renouvellement des fichiers du serveur «Infocrue».

Par ailleurs, $36 \%$ des propriétaires-occupants se déclarent prêts à investir contre seulement $13 \%$ des locataires. D'ailleurs dans les motifs de refus d'investir, les locataires invoquent en premier lieu (44\%) le fait que les mesures de mitigation sont de la responsabilité des propriétaires. Aussi dans la réflexion sur les motivations des personnes à protéger leurs biens il faut d'abord partir de l'enjeu à protéger et non de l'aléa auquel il est exposé. Parmi les propriétaires-occupants, un pourcentage non négligeable a déclaré ne pas être prêt à investir car sur le point de quitter le logement. Ce sont en majorité des personnes âgées. La perspective de devoir quitter le logement dans peu de temps n'incite pas à effectuer des travaux de protection.

Si l'on devait dresser le portrait type de la personne disposée à mettre en place des mesures de protection individuelles contre l'inondation, elle aurait le profil d'un adulte propriétaire de son logement depuis assez peu de temps et ayant vécu une inondation.

\section{LE CAS DIFFICILE DES COMMERCES}

Les commerces ont été enquêtés séparément des logements même si une grande partie du diagnostic était commune. Ils se sont révélés être des biens particulièrement difficiles à adapter pour des raisons techniques et humaines.

\section{Le risque inondation : une préoccupation secondaire}

La vulnérabilité des commerces se lit au travers de deux critères majeurs : d'une part, la vulnérabi- lité intrinsèque face à l'inondation et d'autre part le désintérêt des commerçants pour les mesures d'adaptation. 109 des 194 commerces diagnostiqués n'ont pas accès à un niveau supérieur ce qui empêche toute possibilité de mettre le stock et le matériel d'exploitation à l'abri. Le risque est connu des commerçants puisque $87 \%$ d'entre eux affirment que leur commerce (marchandise ou matériel d'exploitation) est vulnérable à l'inondation mais seulement $20 \%$ ont mis en place des mesures de protection ou d'adaptation aux inondations dans leur commerce. La figure 8 (planche III) montre à l'échelle d'un quartier les différentes attitudes des commerçants face à la prise de mesures d'adaptation du bâti. Le plus souvent, la mesure adoptée consiste en la surélévation du matériel y compris la surélévation temporaire en cas d'alerte; la seconde mesure mise en place est le batardeau. Les commerces les mieux adaptés sont ceux de la rue Madec et de la place de la Terre-au-Duc soumises aux remontées d'eau lors des marées de vives eaux. Le souhait de mettre en place de nouvelles mesures de réduction de la vulnérabilité de leur commerce est évoqué positivement par $21 \%$ des commerçants interrogés. Ce taux ne varie pas entre propriétaires ou simples gérants $\left(\mathrm{KHI}^{2}=0,04\right.$; seuil critique de significativité $=84 \%$ !).

Le fait d'avoir vécu l'inondation de 2000 est déterminant dans le sentiment de menace (tableau 6). Ceci se répercute sur la mise en place de protections. $41 \%$ des commerçants ayant vécu 2000 ont pris des mesures de réduction de la vulnérabilité du commerce ou du stock contre $5 \%$ seulement des commerçants n'ayant pas vécu les inondations de 2000 .

\section{Comment expliquer ce désintérêt?}

Ce désintérêt des commerçants pour la réduction de la vulnérabilité des commerces peut s'expliquer par plusieurs facteurs. Un bon nombre de

\begin{tabular}{|l|c|c|c|}
\hline Critère & Non inondés en 2000 & Inondés en 2000 & Moyenne \\
\hline Sentiment de menace & $46 \%$ & $62 \%$ & $52 \%$ \\
\hline Envisagent des aménagements, prêts à investir & $23 \%$ & $15 \%$ & $20 \%$ \\
\hline Déjà des mesures mises en place (stock et/ou matériel) & $5 \%$ & $41 \%$ & $20 \%$ \\
\hline
\end{tabular}

Tableau 6 : Attitude des commerçants vis-à-vis des mesures de protection Shop-keepers and adaptation measures 
commerces sont en difficulté économique ce qui relègue au second plan les préoccupations moins immédiates. Ces difficultés s'expliquent par le contexte général (concurrence d'internet, crise économique) et local (problème d'accès au centre-ville, développement des commerces en périphérie de la ville...). L'autre facteur est incontestablement le renouvellement rapide des gérants et propriétaires de commerces. $66 \%$ des commerçants se sont installés dans ce commerce après 2001 et $36 \%$ après 2010 (enquête fin octobre 2013). 73 \% de commerçants ne sont pas propriétaires. Cependant, parmi les commerçants qui n'occupaient pas en 2000 le commerce qu'ils occupent actuellement, une grande partie habitait déjà Quimper. Globalement, $71 \%$ des commerçants ont subi la crue de 2000 soit dans leur commerce actuel soit dans un autre commerce. La difficulté de mettre en place des mesures de protection immédiate (élévation du stock, pose de batardeau...) est renforcée par le fait que $87 \%$ des commerçants ne résident pas sur place. Ainsi on constate une certaine contradiction entre, d'une part, une forte conscience du risque et, d'autre part, une faible implication dans la prévention individuelle. Ce n'est donc pas forcément l'absence de connaissance de l'aléa qui conditionne les actions de prévention mais l'absence de volonté d'investissement pour un commerce dont ils ne sont pas propriétaires et qu'ils savent ne pas devoir occuper encore plus de quelques années. Toutefois, $25 \%$ des commerçants se disent prêts à mettre en place des mesures de réduction de la vulnérabilité du commerce et, comme pour les logements, les candidats à l'adaptation se trouvent dans les commerçants propriétaires, installés depuis plus de 10 ans et ayant subi une inondation.

\section{LE CIBLAGE DES MESURES : DU « COUSU-MAIN »}

Ainsi à la lumière de l'enquête menée à Quimper, quels sont les prérequis nécessaires à la mise en place de mesures de réduction de la vulnérabilité du bâti privatif?

\section{Mesures de réduction de la vulnérabilité et aléa inondation}

Les nombreux guides de préconisation rappellent le nécessaire discernement préalable à toute mise en place de mesures de protection individuelles. Il faut en la matière s'adapter au type d'aléa (fréquence, durée de la submersion, hauteur d'eau...), au type de bâti existant, à la population et surtout assortir les mesures techniques des comportements adéquats.

Globalement, les mesures que nous avons rencontrées sur le terrain de l'enquête (relèvement du mobilier, batardeau...) sont efficaces pour des hauteurs d'eau inférieures à un mètre dans le bâtiment. Dans les submersions supérieures à $1 \mathrm{~m}$, la priorité est à la mise en sécurité des personnes. La présence d'un étage ou au moins d'un refuge (terrasse, sortie de toit...) est nécessaire surtout si la montée des eaux est rapide et ne permet pas l'évacuation préventive.

\section{L'adaptation aux situations locales}

L'enquête a montré la diversité des situations locales même à l'intérieur d'une seule commune en l'occurrence Quimper. D'un quartier à l'autre, le vécu des inondations, l'âge de la population, l'aléa, le type d'habitat... conditionnent la réceptivité de la population aux mesures de protection individuelles. Ceci explique en partie l'échec des préconisations inclues dans les PPRi même lorsque les actions sont préconisées finement en fonction de la hauteur du plancher.

\section{S'appuyer sur un échantillon de personnes volontaires}

158 personnes (sur 290) soit $54 \%$ des particuliers dont 53 personnes dans le quartier de l'hippodrome se sont déclarées prêtes à être recontactées dans le cadre d'une campagne de mise en œuvre de mesures de réduction de la vulnérabilité du bâti. Il existe donc un noyau de personnes sensibilisées et prêtes à adhérer à une démarche de réduction de la vulnérabilité (figure 9 - planche IV). En revanche, penser que tous les logements puissent être adaptés est illusoire à court et moyen terme. 
Envisager de développer la mise en œuvre des mesures de mitigation pas à pas en se fondant sur le volontariat et une démarche d'exemple pédagogique nous paraît plus efficace à long terme que des mesures coercitives. Dans cette optique, il convient de considérer les prescriptions incluses dans les PPR comme « ouvrant possibilité à des subventions » plutôt que comme des obligations qui, à court terme, ne seront pas suivies d'effet.

\section{Travailler l'offre préventive post sinistre}

Plus qu'une connaissance abstraite de l'aléa, le vécu de l'inondation est un élément déterminant dans la volonté de mettre en place des mesures de protection individuelle. La période qui suit une inondation est propice à la mise en place de telles mesures; encore faut-il y être préparé. Reconstruire les logements sinistrés de façon à ce qu'ils soient plus résistants à l'eau ne s'improvise pas, le souci des sinistrés étant de réhabiliter au plus vite. Les artisans peuvent devenir porteurs de l'offre préventive en proposant des améliorations dans les revêtements, dans la remise en état du réseau électrique...

L'enquête de Quimper n'envisageait que le point de vue des particuliers dans la mise en place de mesures d'adaptation du bâti existant à l'inondation. Le déploiement massif de mesures de réduction de la vulnérabilité du bâti demande l'engagement de nombreux acteurs (et financeurs) et l'adaptation de procédures : formation des artisans, adaptation des procédures existantes dans les services de l'État... Ainsi des obstacles et des opportunités existent aussi du côté institutionnel (Vinet, 2010).

\section{Conclusion}

L’approche actuelle de la réduction de la vulnérabilité du bâti en France est technicienne, normative et coercitive. Les plans de prévention des risques préconisent ou imposent des mesures générales calées sur l'aléa : par exemple, les habitations affectés par $80 \mathrm{~cm}$ d'eau lors de la crue de référence devront équiper les ouvertures d'un batardeau. Les plans de prévention des risques peinent à déboucher sur des réalisations concrètes (Chabrol et al., 2014) et les prescriptions restent souvent lettre morte faute d'un suivi par les services de l'État.
L'exemple de Quimper montre les limites d'une approche technicienne normative de la réduction de la vulnérabilité du bâti rendue difficile par la diversité des situations individuelles des bâtiments et de leurs occupants face à l'inondation.

Les obstacles sont nombreux et les inerties très fortes mais à la lumière du cas quimpérois, on peut dégager un certain nombre de règles et de leviers propres à faciliter la mise en place de ces mesures.

Le volet des diagnostics quimpérois présenté ici concernait plus spécifiquement le consentement à effectuer des travaux d'adaptation au risque. La volonté des occupants des logements et des commerces d'adapter leur bâtiment résulte d'un complexe faisceau de facteurs. Les résultats et notamment ceux des commerces montrent tout d'abord qu'elle ne dépend pas seulement du sentiment de menace ni du niveau de connaissance du risque des occupants. Ainsi, penser qu'il suffit pour les autorités de développer la conscience du risque pour déclencher l'adhésion à des mesures d'adaptation du bâti face au risque est sans doute insuffisant. L’hétérogénéité des attitudes par rapport à l'adoption de mesures d'adaptation tient autant à l'attachement au bien qu'à la connaissance de l'aléa. On retrouve là tout le sens de la définition du risque donnée par Metzger et d'Ercole (2009) «le risque c'est la probabilité de perdre ce à quoi l'on tient ». Cette définition est centrée sur l'enjeu et non sur la perception de l'aléa. Sous cet angle, l'amélioration de la conscience du risque est certainement un préalable nécessaire à toute démarche de réduction de la vulnérabilité (Colbeau-Justin et al., 2003) mais elle est loin d'être suffisante. Une communication uniquement fondée sur un martelage de la connaissance de l'aléa n'aurait que peu d'effets. Cette communication pourrait intégrer des éléments de connaissance plus concrets sur les impacts attendus d'une inondation.

L'attachement au bien nous semble être un facteur essentiel motivant les personnes à adapter leur bâti. Il est apparu au cours du dépouillement des enquêtes mais il est complexe à saisir et varie en fonction des individus. Colbeau-Justin et al. (2003) évoque l'attachement au bien relevé lors des destructions suite aux inondations mais ils ne l'associent pas à la volonté de mettre en place des mesures de prévention. Or il nous semble que cette piste est à creuser pour mieux comprendre la pro- 
pension des personnes à vouloir s'impliquer dans la prévention individuelle. Une personne est attachée à son logement et voudra le protéger si elle se projette dans l'avenir dans ce logement. Cela dépend de l'âge, du statut de propriétaire mais pas seulement. Une enquête plus approfondie sur les aspects psychosociologiques serait nécessaire pour préciser les résultats sur cette question.

En attendant, quelles conclusions peut-on tirer pour la mise en place de mesure d'adaptation du bâti ? Plutôt qu'une approche générale et coercitive, il convient, nous semble-t-il, de s'appuyer sur les personnes disposées à investir dans la protection de leur bien en leur démontrant l'intérêt des mesures préventives. Ces personnes identifiées lors de l'enquête constituent $30 \%$ des personnes rencontrées. Il importe aussi de valoriser les bonnes pratiques comme celles rencontrées dans le quartier de l'Hippodrome, relativement bien adapté à l'inondation.

Le consentement à mettre en place des mesures d'adaptation est plus faible pour les commerces que pour les logements. La rotation des propriétaires et des gérants de commerces est rapide (quelques années) et diminue la rentabilité potentielle de mesures d'adaptation.

Globalement, la population quimpéroise (habitants comme commerçants) est bien consciente de son exposition aux inondations mais connait peu les mesures possibles d'adaptation du bâti. Une campagne d'information devrait porter sur ces mesures en même temps que la sensibilisation des professionnels du bâtiment qui sont les pourvoyeurs d'offre préventive souvent oubliés des campagnes de sensibilisation à la prévention des risques.

Dans cette approche très fine de la réduction de la vulnérabilité, seul l'engagement fort des collectivités territoriales peut faire évoluer les choses. Les diagnostics se multiplient mais la réalisation des mesures d'adaptation est encore loin d'être généralisée. Les initiatives positives qui éclosent çà et là requièrent beaucoup d'énergie et une démarche adaptée, pédagogique recherchant l'adhésion des populations.

\section{Bibliographie}

Andjelkovic I., 2001. Guidelines on non-structural measures in urban flood management IHPV/Technical documents in hydrology/ $n^{\circ}$ 50. International hydrological programme. Unesco, 81 p. [http://unesdoc.unesco.org/ images/0012/001240/124004e.pdf] consulté le 15 mars 2015.

André C., 2013. Analyse des dommages liés aux submersions marines et évaluation des coûts induits aux habitations à partir de données d'assurance. Perspectives apportées par les tempêtes Johanna (2008) et Xynthia (2010). Thèse de doctorat de l'Université de Bretagne Occidentale, Brest. 295 p.

Aviotтi A., 2014. Réduire la vulnérabilité de l'habitat individuel face à l'inondation, Paris, Lavoisier Tec \& Doc, coll. «Sciences du risque et du danger », 214 p.

CEPRI, 2010. Le bâtiment face à l'inondation Diagnostiquer et réduire sa vulnérabilité Guide méthodologique, Orléans, Centre européen de prévention du risque d'inondation, 53 p.

Certu-MEDD, 2004. Centre-ville en zone inondable. Prise en compte du risque. Dix exemples d'adaptation du bâti, Paris, ministère de l'Écologie et du Développement Durable, 68 p.

Chabrol D., Bodino Ph., Levendic Ph., Pitie Ch., Barrey G., Brassens B., Le Boulaire Ch., 2014. évaluation à mi-parcours du plan «submersions rapides », Conseil général de l'environnement et du développement durable, inspection générale des finances, inspection générale de l'administration, inspection de la défense et de la sécurité civiles, 155 p.

Colbeau-Justin L, Casal A., Barnay J., 2003. Étude de la vulnérabilité psychologique de l'habitant face au risque d'inondation, Enquête sur les inondations de la Bretagne et de la Saône d'après EDATER, Ledoux consultant, LPE UMR 8069, 69 p.

Crichton D., 2003. Temporary local flood protection in the United Kingdom - An independent assessment, London, Benfield Hazard Research Centre Miscellaneous Papers, University College London.

DGUHC, 2005. Inondations. Guide de remise en état des bâtiments, Direction Générale de l'Urbanisme, de l'Habitat et de la Construction, Ministère de l'Équipement, du Logement et des Transports, $28 \mathrm{p}$.

EPTB SaÔne et Doubs, 2009. Réduction de la vulnérabilité de l'habitat aux inondations. Préconisations techniques. 34 p. [http://www.observatoire-saone.fr/].

FEMA, 1999. Protecting building utilities from flood damagePrinciples and practices for the design and construction of flood resistant building utility systems, Federal Emergency Management Agency, FEMA Publications, Washington, DC. 160 p. + annexes.

Jousseaume, V., Mercier D., 2009. Évaluer la vulnérabilité architecturale de l'habitat en zone inondable : l'exemple du val nantais, in Becerra S., Peltier A. (dir.), Risques et environnement: recherches interdisciplinaires sur la vulnérabilité des sociétés, Paris, L’Harmattan, p. 199-214.

Kelman I., 2002. Physical flood vulnerability of residential properties in coastal areas, eastern england, thèse de doctorat de l'Université de Cambridge, Royaume-Uni 311 p. [http:// www.ilankelman.org/phd.html] (consulté le 15 mars 2015). 
Kelman I., 2007. Decision-making for flood-threatened properties in S. Begum, Stive MJF, Hall JW (eds.). Flood Risk Management in Europe, Springer, Dordrecht, p. 3-19.

Kreibich H., Thieken A. H., Petrow Th., Müller M., Merz B., 2005. Flood loss reduction of private households due to building precautionary measures - lessons learned from the Elbe flood in August 2002. Natural Hazards and Earth System Sciences, 5, p. 117-126.

Leone F., Vinet F. (dir.), 2014. Diagnostic et mesures de réduction de la vulnérabilité face aux inondations pour les logements et commerces de la ville de Quimper - Rapport collectif de stage de terrain, Master 2 « Gestion des Catastrophes et des Risques Naturels », Département de Géographie, Université Paul Valéry - Montpellier 3, 175 p. + Annexes (atlas cartographique, BD SIG).

MEDDE, 2011 . Première évaluation nationale des risques d'inondation. Principaux résultats EPRI 2011.16 p. [http://www. developpement-durable.gouv.fr/-Gestion-des-risques-d-inondations-.html].

MEDDE, 2012. Référentiel de travaux de prévention du risque inondation dans l'habitat existant, 91 p. [http://www.developpement-durable.gouv.fr/Referentiel-de-travaux-de.html].

MEEDDAT, 2008. Quinze expériences de réduction de la vulnérabilité de l'habitat aux risques naturels. Retours d'expériences, Risques naturels majeurs : Quels enseignements?, 36 p.

Metzger P., D'Ercole R., 2009. Enjeux territoriaux et vulnérabilité : une approche opérationnelle, in BECERRA S., Peltier A. (dir.), 2009. Risques et environnement: recherches interdisciplinaires sur la vulnérabilité des sociétés, Paris, L'Harmattan, 575 p.

MEDD, 2004. Guide pratique pour la prise en compte de la vulnérabilité des bâtiments existant dans les P.P.R. inondations, ministère de l'Écologie et du Développement Durable, 47 p.
Salagnac J.-L., Bessis B., 2006. Réduire la vulnérabilité des bâtiments en zones inondables, in LEONE F., Vinet F. (dir.) La vulnérabilité des sociétés et des territoires face aux menaces naturelles. Analyses géographiques, vol. 1, Montpellier, université Paul-Valéry, coll. «Géorisques », p. 119-123.

Salagnac J.-L., Hand D., Florence C., Delpech P., Axès J.-M., 2014. Impacts des inondations sur le cadre bâti et ses usagers, rapport final CSTB, $47 \mathrm{p}$.

Sivalodet, 2012. Programme d'Actions de Prévention des Inondations (PAPI) - Bassin de l'Odet 2012/2017 - 39 p. + annexes [www.sivalodet.fr/].

Tовтевотот J.-P., 1993. Le coût des dommages dus aux inondations : estimation et analyse des incertitudes, Thèse de l'ENPC-CERGRENE, Noisy-le-Grand, 287 p. + annexes.

Thieken A. H., Müller M., Kreibich H., Merz B., 2005. Flood damage and influencing factors: New insights from the August 2002 flood in Germany, Water Resour. Res., 41, W12430, doi:10.1029/2005WR004177.

VALY J., 2010, Croissance urbaine et risque inondation en Bretagne, Thèse de doctorat, Université Rennes 2, 525 p. + annexes, [http://tel.archives-ouvertes.fr/docs/00/62/46 /46/ PDF/theseValyJanique.pdf].

Verrhiest G., Guézo B., 2006. Intégrer la construction dans son milieu physique, Préventique-sécurité, n 89, p. 37-42.

Vinet F., 2010. Le risque inondation. Diagnostic et gestion, Paris, Lavoisier Tec\&Doc, coll. «SRD », 232 p.

[http://www.alabri-smage.fr/] site dédié de l'opération de réduction de la vulnérabilité du bâti dans le département du Gard. 